\title{
Potential errors in the estimation of coronary arterial stenosis from clinical arteriography with reference to the shape of the coronary arterial lumen
}

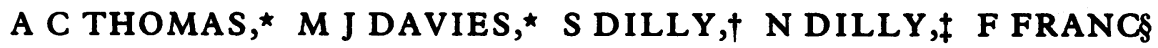

From the Department of Histopathology, ${ }^{\star}$ British Heart Foundation Cardiovascular Unit and $\ddagger$ Department of Anatomy, St George's Hospital Medical School, London; †Department of Histopathology, Royal Marsden Hospital, Sutton; and §Institute of Physics, Czechoslovak Academy of Science, Prague, Czechoslovakia

SUMMARY Underestimation of coronary arterial stenosis at clinical arteriography has been explained by inadequate radiological views of crescentic or slit-shaped lumens. Postmortem perfusion-fixation of the coronary arteries at physiological pressures shows that most stenotic lesions result in circular, elliptical, or D-shaped lumens. Crescentic lumens in fully distended vessels are associated only with acute mural thrombus projecting into the lumen or with massive intra-intimal thrombus and plaque fissuring. Elliptical and D-shaped lumens, however, adequately explain the clinically observed phenomenon that some stenotic lesions can be seen only in one $x$-ray plane and not in another. It is no longer correct to postulate a crescentic or slit-like lumen. Furthermore, not only can elliptical and D-shaped lumens result in underestimation of stenosis, they may also result in serious overestimation. Lumens need only to deviate slightly from being circular for error to be introduced into the estimation of stenosis by currently used methods. The calculation of percentage stenosis from densitometric measurements or from computerised reconstruction of the arterial lumen is, however, more accurate.

The estimation of coronary arterial stenosis from clinical arteriography is usually made by comparing the diameter of the coronary lumen at the point of stenosis with that of an adjacent presumably normal arterial segment in the radio-opaque contrast filled artery (percentage stenosis by diameter). It is widely accepted that such measurements may underestimate the degree of arterial stenosis if one plane only is available for examination. The usual explanation given is that the residual coronary arterial lumen is slit-like or crescentic in shape. ${ }^{12}$ These explanations derive from widespread illustration of irregularly shaped lumens in standard textbooks and from the common belief that atheromatous plaques bulge into the lumen. Histological sections depicting such irregular shapes are, however, obtained from material that has been

Requests for reprints to Dr A C Thomas, Histopathology Department, St George's Hospital Medical School, Cranmer Terrace, Tooting, London SW17 ORE.

Accepted for publication 1 October 1985

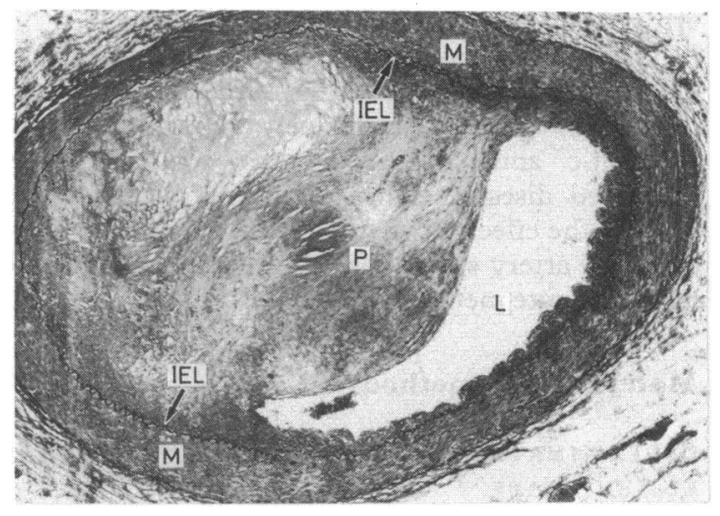

Fig. 1 Histological cross section of atherosclerotic coronary artery fixed in the undistended state. The atheromatous plaque $(P)$ appears to project into the lumen $(L)$ and this results in an apparently crescentic lumen. The wavy outline of the internal elastic lamina (IEL) indicates fixation in the undistended state. $M$, media. Elastic haematoxylin and eosin $\times 45$ (original magnification). 


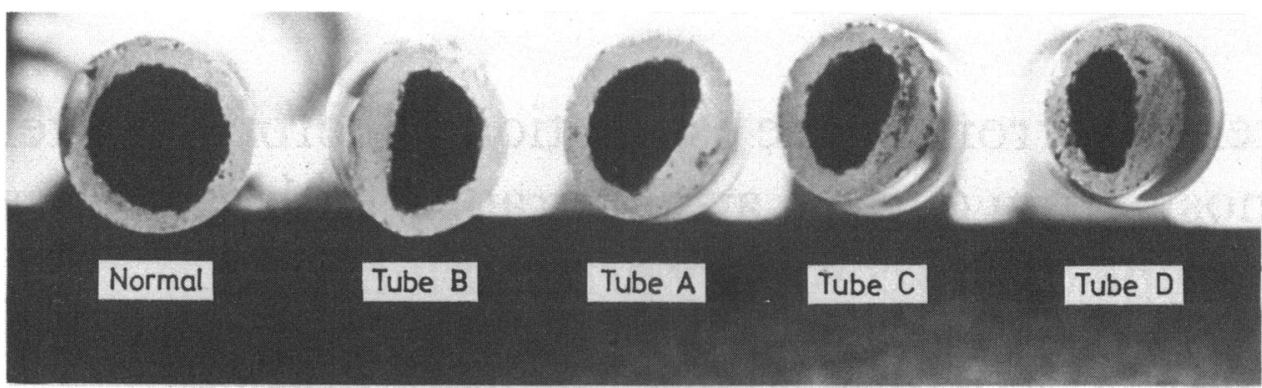

Fig. 2 Cross sections of four manipulated glass tubes ( $A$ to D) transected at maximum points of stenoses to show residual ovoid lumens compared with cross section of normal unmanipulated glass tube with circular lumen.

fixed and processed in the undistended state (Fig. 1). Crawford and Levene in 1953 showed that the "raised fibrofatty plaque" was somewhat of a misnomer and that though atheromatous plaques in arteries fixed in complete distension reduced luminal cross sectional area they did not project into the arterial lumen as a hump with well defined margins but appeared en face as changes in consistency and colour. ${ }^{3}$ Other workers later confirmed this finding and concluded that most diseased arterial segments had circular lumens and that crescentic lumens were rarely seen. ${ }^{4-7}$ Percentage stenosis in histological sections is usually calculated from vessel and lumen perimeters assuming these to be circumferences of circles in calculating cross sectional area. This method overcomes any distortion introduced by undistended vessels and in the production of a histological section but might introduce important errors if indeed the lumens are not circular.

The objects of the present study were to investigate the shape of the diseased coronary artery lumen, to prove conclusively whether or not crescentic and slit-like lumens occur in fully distended diseased coronary arterial segments, to observe the effects of luminal shape on estimation of coronary artery stenosis, and to explore alternative more accurate methods for such estimation.

\section{Materials and methods}

DETERMINATION OF THE CORONARY ARTERIAL LUMEN SHAPE

The hearts of nine patients who died of ischaemic heart disease were perfused-fixed at either 100 or $150 \mathrm{~mm} \mathrm{Hg}$ with $10 \%$ formol saline by tying a large cannula into the aortic root. Under pressure the normal aortic valve closes and the coronary arteries are perfused-fixed in the fully distended state. ${ }^{8}$ After perfusion-fixation for 24-48 hours, postmortem angiography was carried out by injecting a warm suspension of $5 \%$ gelatin/barium sulphate at a pressure corresponding to the perfusion-fixation pressure and allowing this to solidify within the coronary lumen. This technique allowed localisation of stenotic lesions by $x$-ray examination and supported the arterial wall during the subsequent processing to produce histological sections, a process which may cause considerable distortion of lumen shapes in undistended vessels. In each heart the epicardial coronary arterial tree was dissected en bloc with surrounding adipose tissue and decalcified for $\mathbf{7 2}$ hours in $10 \%$ formic acid. After decalcification the coronary arteries were transversely sectioned every $3 \mathrm{~mm}$ throughout their lengths; we ensured that the transverse sections were perpendicular to the longitudinal axis of the artery. Selected segments were examined and

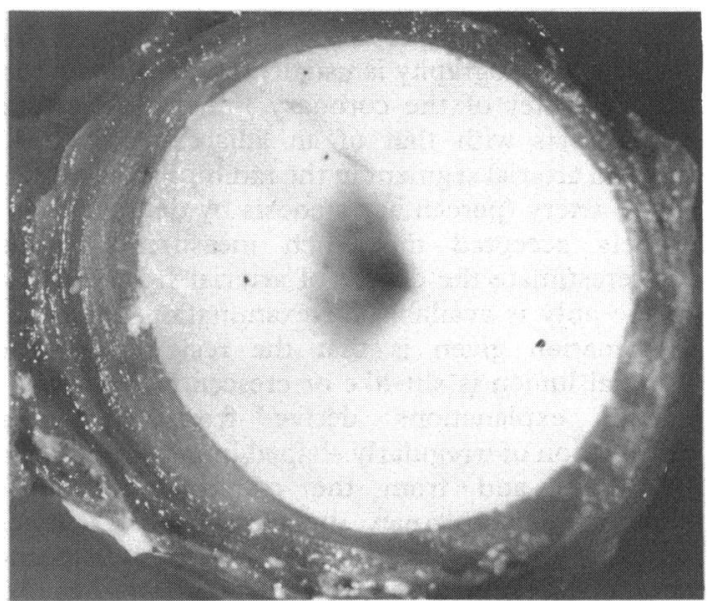

Fig. 3 Appearance under the dissecting microscope of a cross section of normal coronary artery perfused-fixed at $150 \mathrm{~mm} \mathrm{Hg}$. Both the external shape and internal barium filled lumen are almost perfectly circular. $\times 25$ (original magnification). 


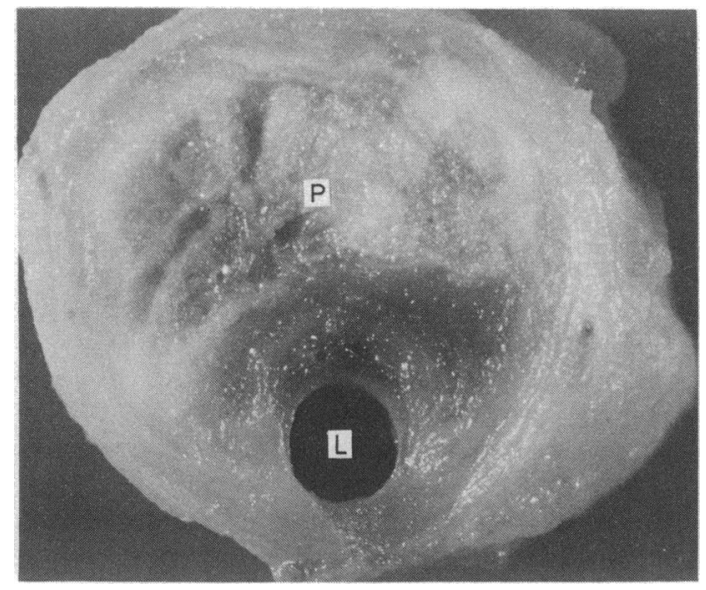

Fig. 4 Appearance under dissecting microscope of cross section of atherosclerotic coronary artery perfused-fixed at $150 \mathrm{~mm} \mathrm{Hg}$. The residual lumen $(L)$ approximates to being a circle although it is eccentrically positioned. $P$, atheromatous plaque. $\times 25$ (original magnification).

photographed (without further preparation) under the dissecting microscope. Histological sections of each paraffin embedded $3 \mathrm{~mm}$ segment were prepared, stained with an elastic haematoxylin and eosin technique, examined under the light microscope, and compared with the corresponding gross appearance under the dissecting microscope.

\section{INVESTIGATION OF EFFECT OF LUMEN SHAPE ON OBSERVED PERCENTAGE STENOSIS Computer simulation}

A BBC Model B microcomputer (Acorn Co.) was programmed to construct a simulation of a cross section of coronary artery in which the vessel outline is circular but the stenosed lumen may be chosen as a circle, ellipse, or D shape and where the D shape may be formed of two half ellipses. (The term "ovoid" is used to include ellipses and all D shapes.) The program allows a full range of choice of the position of the centre of the lumen, vertical and horizontal diameters, and $x$-ray beam incidence. It then offers a print out of $(a)$ the observed percentage stenosis by diameter; $(b)$ the observed percentage stenosis by cross sectional area, assuming the observed lumen and vessel widths are diameters of circles; and $(c)$ the true percentage stenosis by cross sectional area. The program can then produce graphs of the observed percentage stenosis as diameter or cross sectional area measurements against degrees of rotation of the incident $x$-ray beam. A similar graph of lumen position and observed lumen width against degrees of $x$-ray rotation can also be obtained. Finally, for any given $x$-ray beam incidence the program will plot a densitometric readout of the vessel lumen. From the shapes and positions of such densitometric readouts in various $x$-ray planes predictions about the size and shape of the actual lumen may be made.

\section{Experimental study}

Artificial stenoses of varying severity were created in glass tubes by manipulating the heated glass to give residual ovoid lumens (Fig. 2). The glass tubes were filled with a $5 \%$ gelatin/barium sulphate suspension and radiographs were subsequently obtained through different degrees of rotation in the horizontal plane when the $x$-ray beam was perpendicular to the long axis of the tubes. The tubes were rotated through varying degrees in the vertical plane when the $x$-ray beam was no longer perpendicular to the long axis of the tubes, and this was also performed for different degrees of rotation in the horizontal plane. The observed percentage stenoses by diameter and corresponding cross sectional area, assuming the observed lumen and vessel widths to be diameters of circles, were calculated for each position. The true percentage stenosis of the cross sectional area was measured by planimetry from cross sections of the glass tubes.

\section{Results}

\section{Determination of coronary arterial lumen shape}

Normal arterial segments which have been perfused-fixed in the fully distended state at physiological pressures have both a circular external shape and a circular lumen when viewed at right angles to the arterial longitudinal axis (Fig. 3). The corresponding histological sections show the internal elastic lamina to be smoothed out, indicating that the artery has indeed been fixed in a state analogous to full dilatation in life. Any deviation from a circular lumen seen in histological sections is a result of distortion at the time of preparing the sections and is a purely technical artefact. Cross sections that are not made perpendicular to the longitudinal arterial axis will result in ovoid shaped lumens when they are viewed end on at right angles to the plane of cut. The external shape of the artery, however, will also be ovoid.

In atherosclerotic arterial segments most of the lumens approximated to being circular but are often placed eccentrically to the midline of the vessel (Fig. 4). Elliptical and D-shaped lumens are seen in diseased segments viewed perpendicularly to the longitudinal arterial axis in hearts perfused-fixed at $100 \mathrm{~mm} \mathrm{Hg}$ and $150 \mathrm{~mm} \mathrm{Hg}$ (Figs. 5a, b, and c). 


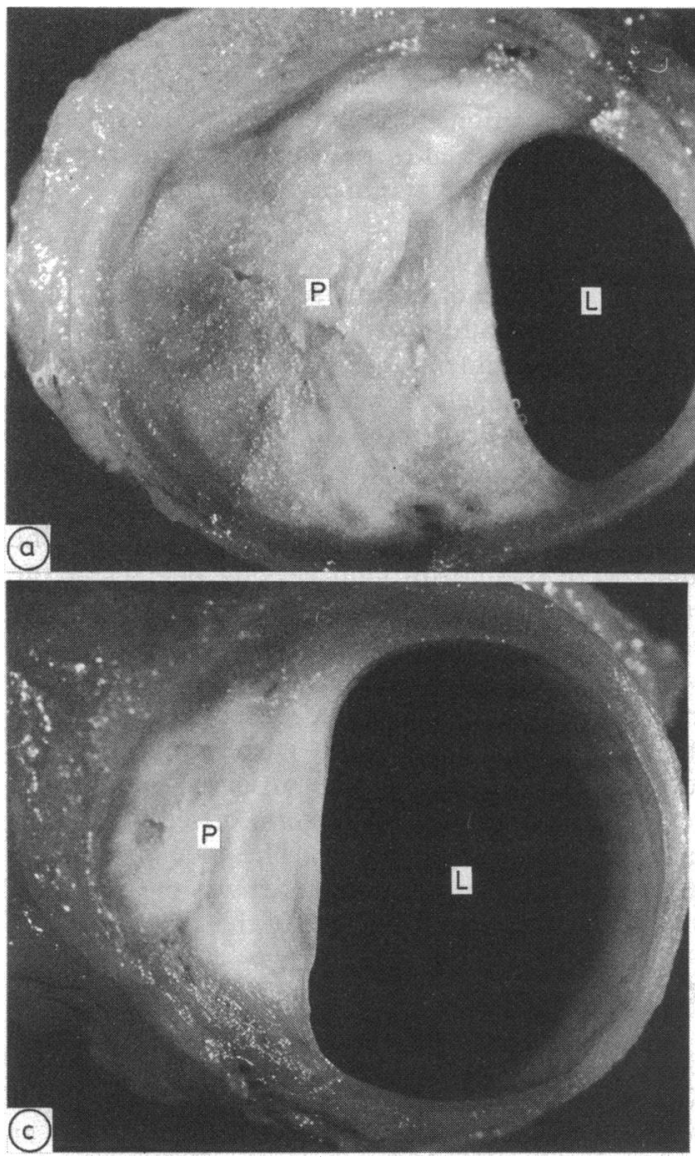

The external shape of the artery is often ovoid as a result of the atheromatous plaque bulging outward into the media and is often associated with focal destruction of the internal elastic lamina. The corresponding histological sections confirm complete distension as judged by a smooth internal elastic lamina where present (Fig. 6). The lumen appears to be flattened over the fibrous cap of a plaque, which is often associated with an underlying lipid-rich pool. Not all lipid-rich plaques give rise to elliptical or D-shaped lumens; some are associated with circular lumens. Most hard fibrous plaques without lipid-rich pools, however, result in circular lumens. Slit-like and crescentic lumens do occur, but only with rapidly evolving lesions in which blood from the lumen has tracked into the atheromatous plaque via a fissure and this has led to massive intra-intimal thrombosis or in which a polypoidal mass of thrombus projects into the lumen (Figs. 7a and b). Crescentic and slit-like lumens are not a feature of atheromatous plaques
Fig. 5 Appearance under dissecting microscope of cross sections of atherosclerotic coronary arteries perfused-fixed at $150 \mathrm{~mm} \mathrm{Hg}$ showing (a) residual eccentrically positioned elliptical lumen, (b) residual centrally positioned elliptical lumen, and (c) residual eccentrically positioned D-shaped lumen. L, lumen; $P$, atheromatous plaque. All sections $\times 25$ (original magnification).

uncomplicated by acute thrombosis and plaque fissuring.

\section{INVESTIGATION OF EFFECT OF LUMEN SHAPE ON OBSERVED PERCENTAGE STENOSIS \\ Computer simulation}

The computer program shows that for a coronary artery lumen that is circular there is no variation in the observed degree of stenosis for different rotational $x$-ray planes, though the observed position of the stenosis will change if the lumen is eccentrically placed within the vessel.

Ovoid lumens, which are known to occur in fully distended atherosclerotic arteries, show considerable variation in the degree of observed stenosis depending upon the rotational $x$-ray plane. Calculation by area of the observed stenosis can both overestimate and underestimate the true stenosis (Fig. 8), but underestimation will occur by a greater amount and for a wider range of incident $x$-ray beam angles. This is because an elliptical lumen with 


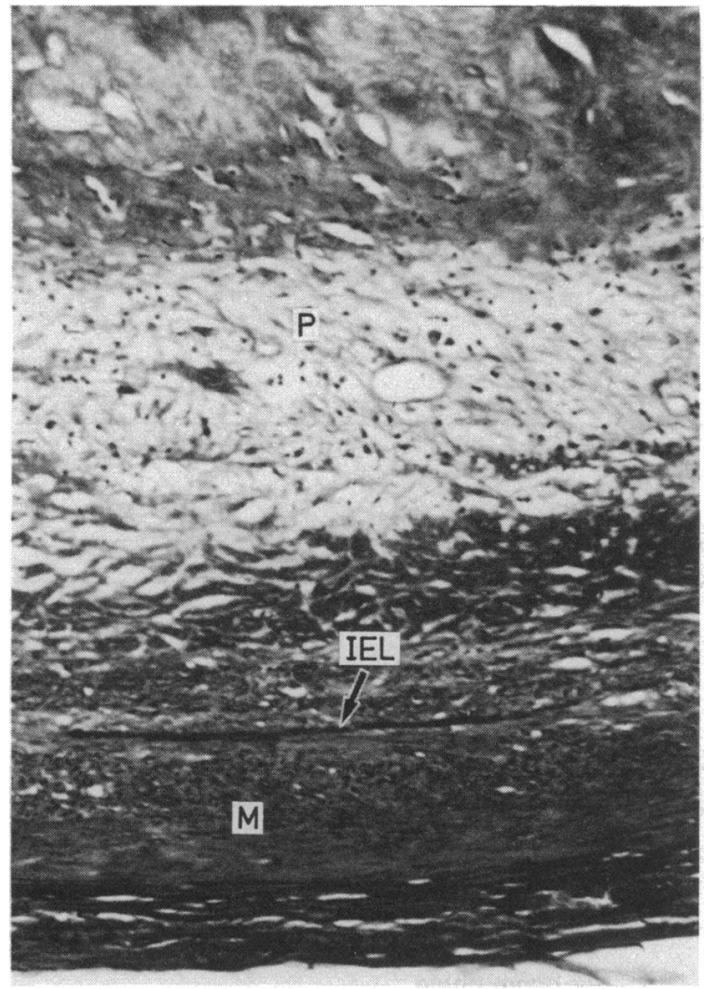

Fig. 6 Histological section of atherosclerotic coronary artery perfused-fixed in full distension as shown by smooth internal elastic lamina (IEL) (see Fig. 1). P, atheromatous plaque; $M$, media. Elastic haematoxylin and eosin $\times 180$ (original magnification).
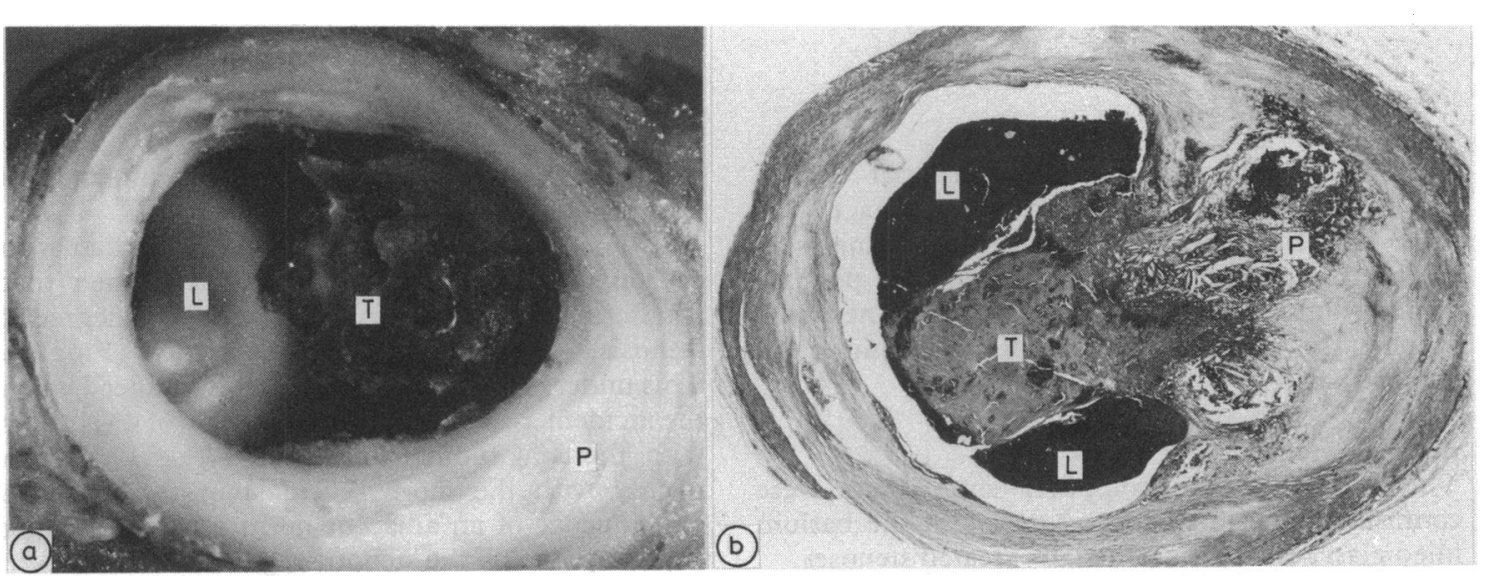

Fig. 7 Cross section of atherosclerotic coronary artery perfused-fixed at $150 \mathrm{~mm} \mathrm{Hg}$ showing residual partly barium filled crescentic lumen $(L)$ produced by projection of polypoidal mass of acute thrombus $(T)$ into arterial lumen. $P$, atheromatous plaque. (a) Appearance under dissecting microscope ( $\times 25$ (original magnification) ). (b) Corresponding histology and appearance under light microscope $(\times 30$ (original magnification)).

principal radii $A$ and $B$ will have a true area of $\pi A B$ whereas the calculated circular area will range from $\pi \mathrm{A}^{2}$ to $\pi \mathrm{B}^{2}$. The correct area will be calculated when the measured radius $(M)$ equals $\sqrt{\mathrm{AB}}$ and, hence, $\pi \mathrm{M}^{2}=\pi \mathrm{AB}$. The square root of $\mathrm{A} \times \mathrm{B}$ will always be closer to the smaller radius and so luminal area will be more severely overestimated, thus producing a greater error in underestimating percentage stenosis.

Varying the principal radii while maintaining a constant area influences both maximum overestimates and underestimates and also the range of incident $x$-ray beam angles for which over and under estimal es will occur (Fig. 8b).

For luminal shapes with the same maximum and minimum diameters the range of incident $x$-ray beam angles for which the observed stenosis is underestimated also depends on the shape of the lumen (Fig. 8c). The graph of calculated stenosis area against beam angle gives an approximate sine wave for an ellipse but not for a D shape. Therefore, although a D-shaped lumen will result in a maximum under and over estimate of stenosis identical to that of an ellipse, a greater percentage of beam angles will result in an underestimate than with the elliptical lumen.

For any given $x$-ray beam incidence the area covered by the densitometric luminal readout will reflect exactly the true luminal cross sectional area irrespective of the shape of the lumen. If this can be related to a similar densitometric readout of a normal segment of artery, then densitometry will provide a true percentage measurement of cross sectional area stenosis (Fig. 9). In order to predict 

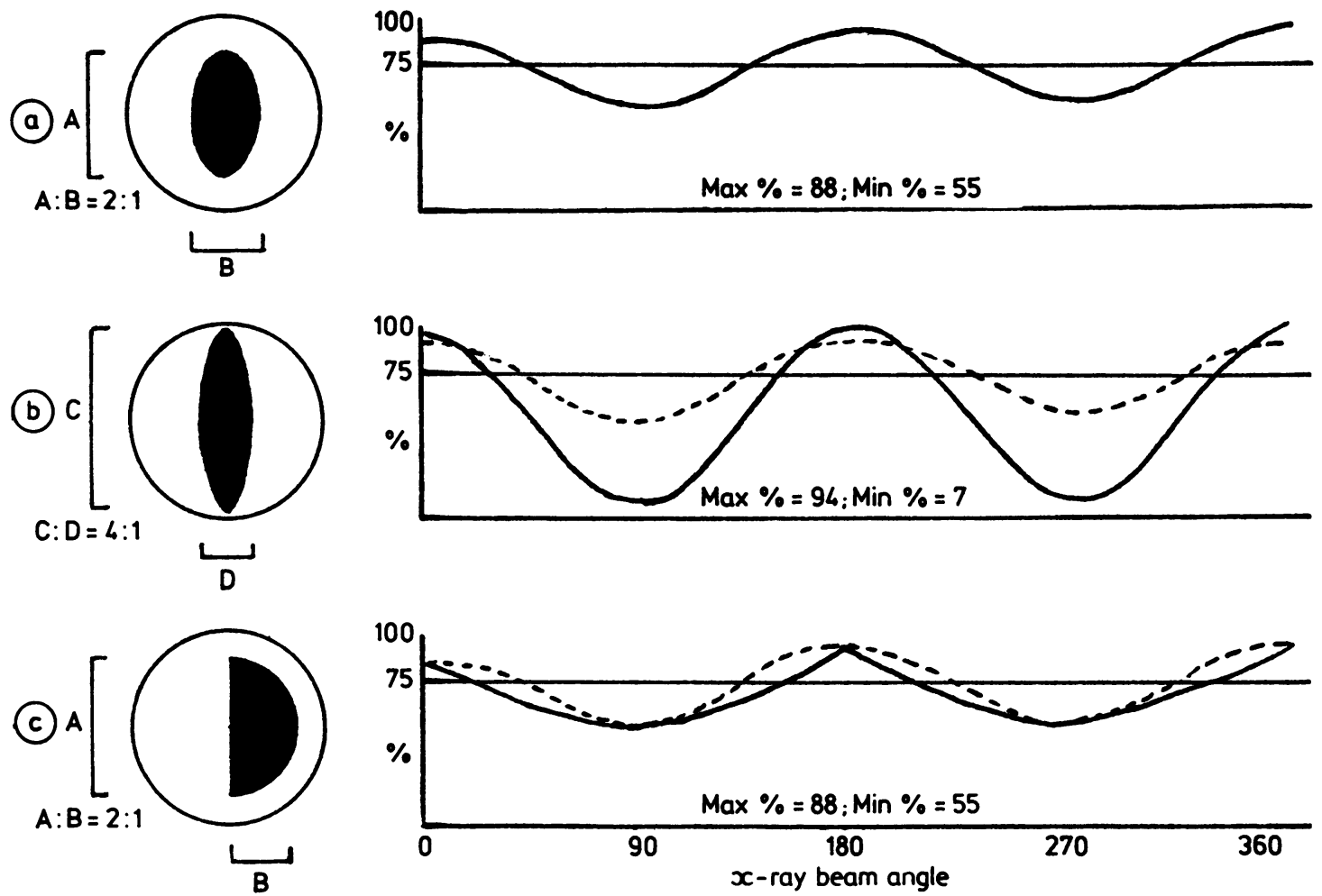

Fig. 8 Graphs in which calculated stenosis area is plotted against $x$-ray beam angle. Each of the lumens has a true stenosis area of $75 \%$ of the maximum vessel area as indicated by the horizontal line on the graph. (a) The ratio of the principal diameters (and therefore radii). A:B is $2: 1$ of the central elliptical lumen. (b) The ratio $C: D$ is now $4: 1$. This greatly increases the maximum and minimum estimation error and also increases the range of beam angles at which stenosis is underestimated. (c) The principal diameters $A$ and $B$ are the same as for (a). This illustrates the effect of lumen shape on the range of beam angles at which stenosis is underestimated. Dotted line in b and cindicates superimposed graph from (a).

the shape and position of the arterial lumen, however, different densitometric readouts of the same stenotic lumen at different known angles of rotation are necessary. Limiting the possible stenotic luminal shapes to those found at necropsy reduces the number of $x$-ray studies required for reconstruction.

\section{Experimental study}

The results of the computer simulations were confirmed experimentally by means of the barium filled glass tubes with artificially created stenoses.

Figure 10 shows the effect of observed stenosis of rotating a residual ovoid shaped lumen through varying degrees in the horizontal plane. The observed cross sectional area of stenosis may vary between $36 \%$ and $82 \%$ (equivalent to $20 \%$ and $58 \%$ by diameter) for a true fixed cross sectional area stenosis of $58 \%$.

If the incident $x$-ray beam is not perpendicular to the longitudinal axis of the barium filled lumen (that is differing degrees of vertical rotation) the degree of stenosis will be further underestimated (Fig.11). This underestimation will only be pronounced when the incident $x$-ray beam forms an angle of $45^{\circ}$ or less with the longitudinal axis of the vessel, is seen mainly with the more severe stenoses, and is a consequence of an adjacent normal segment being superimposed on the stenotic segment.

\section{Discussion}

During the investigation of coronary artery disease by postmortem angiography we became aware that 

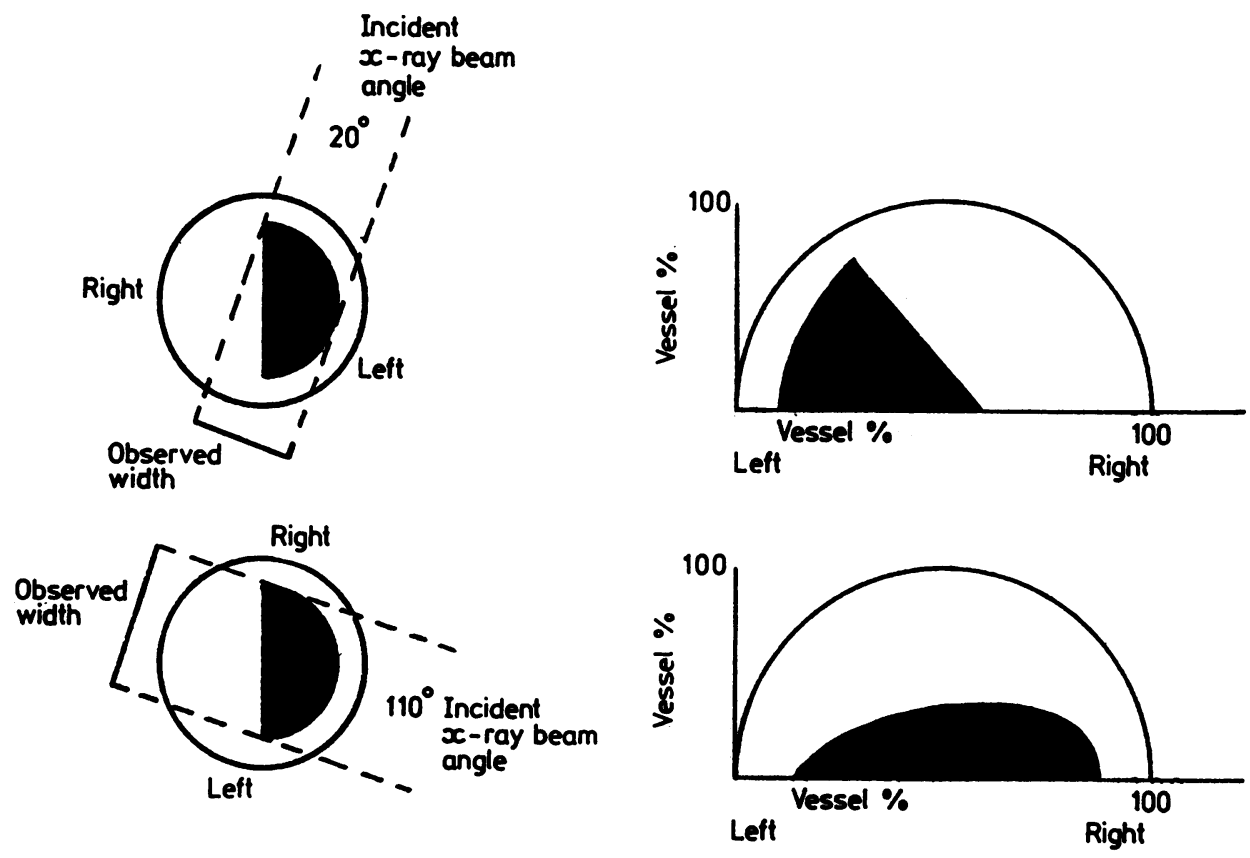

Fig. 9 Densitometric readings for a D-shaped lumen with a true stenosis area of $75 \%$ at beam angles of $20^{\circ}$ and $110^{\circ}$. The solid black areas represent the stenosed lumen and corresponding densitometric recording. The area within the continuous line represents the maximum recording for non-stenosed vessel. The densitometric scan proceeds in the direction of the $x$-ray beam from left to right. Whatever the lumen shape or $x$-ray beam angles comparison of the stenosed and non-stenosed regions will give a true percentage stenosis area.

slit-like and crescentic shaped lumens were rare. In our previous studies postmortem coronary angiography was carried out by injection of a warm suspension of $5 \%$ gelatin/barium sulphate into the coronary ostia of the fresh intact heart at a monitored pressure of $100 \mathrm{~mm} \mathrm{Hg}$ and ligation of the coronary arteries on withdrawal of the cannula. ${ }^{9}$ The gelatin/barium suspension set solid within the epicardial coronary arterial tree and the heart was fixed intact in $10 \%$ formol saline so that the arteries were fixed in distension. Subsequent histological sections of these barium filled coronary arteries after decalcification showed that the residual lumen in diseased segments approximated to being circular, although it was often eccentrically placed, and that crescentic shaped lumens were only encountered in association with plaque fissuring and thrombosis. These observations were in general agreement with those of Freudenberg and Lichtlen who, using a similar technique for postmortem angiography, found that $74 \%$ of all significantly stenosed vessels had eccentric lumens and $26 \%$ central lumens. ${ }^{10}$ Ninety five per cent of all lumens in their cases approximated to being circular, elliptical, or
D-shaped while only 5\% showed irregular star-shaped or crescentic lumens. With postmortem angiography alone and use of a viscous injection medium, however, it is difficult to ensure complete distension of vessels even when the monitored injection pressure is as high as $150 \mathrm{~mm} \mathrm{Hg}$. Certainly a vessel beyond a severe stenosis may be filled at a much lower pressure than that actually monitored and this might account for a few examples of irregular star-shaped lumens observed by Freudenberg and Lichtlen. Furthermore, even in non-diseased arteries that were fully distended (as judged by the histological appearance of a smoothed out internal elastic lamina) the lumen was sometimes distorted as a result of technical problems encountered in the production of a histological section.

The present study ensured fixation of arterial lumens in complete distension by perfusion-fixation of the intact coronary arteries at a constant and monitored perfusion pressure of either 100 or $150 \mathrm{~mm} \mathrm{Hg}$ with low viscosity formol saline. Examination of cross sections of coronary arterial segments with the dissecting microscope before 
Fig. 10 Radiographs of barium filled glass tubes ( $A$ to $D)$ with artificially created stenoses of varying severity and resultant elliptical lumens (see Fig. 2). P'ercentage stenoses by diameter and corresponding cross sectional area at maximum point of constriction were calculated from diameters observed through varying degrees of horizontal rotation and compared with true cross sectional area stenosis measured by planimetry. $x$-ray beam $90^{\circ}$ to longitudinal axis of tubes. (a) Optimum rotational plane to give maximum observed stenosis. (b) Rotational plane $90^{\circ}$ to optimum plane.
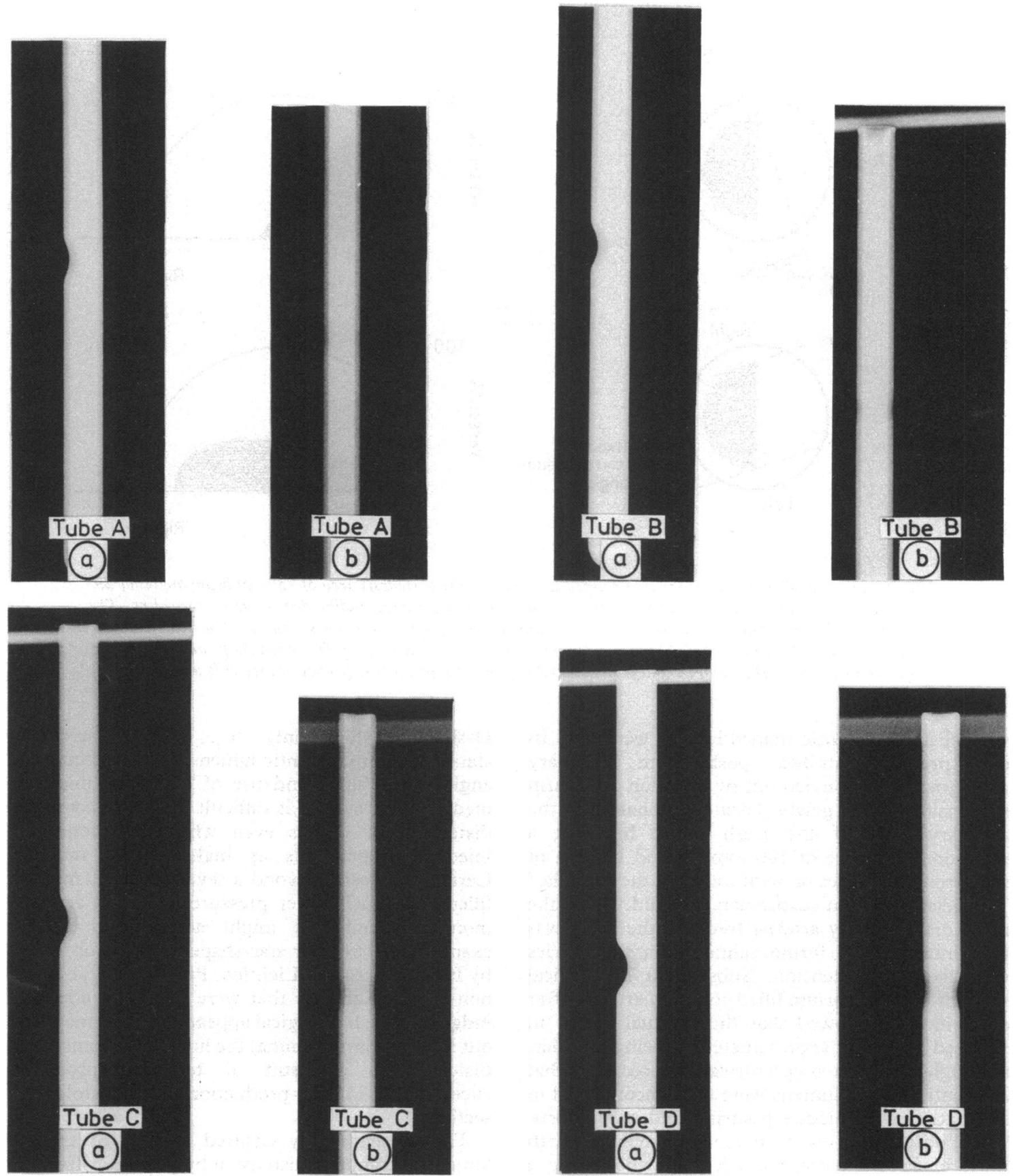

Degrees rotation

\begin{tabular}{lllllllll}
\hline Degrees rotation & \multicolumn{2}{c}{ Tube A (\% stenosis) } & \multicolumn{2}{c}{ Tube B (\% stenosis) } & \multicolumn{2}{c}{ Tube C (\% stenosis) } & \multicolumn{2}{c}{ Tube D (\% stenosis) } \\
\cline { 2 - 9 } & $D$ & $A$ & $D$ & $A$ & $D$ & $A$ & $D$ & $A$ \\
\hline 0 & 20 & 36 & 30 & 51 & 36 & 59 & 56 & 81 \\
20 & 30 & 51 & 36 & 59 & 46 & 71 & 58 & 82 \\
40 & 30 & 51 & 28 & 48 & 40 & 64 & 50 & 75 \\
60 & 18 & 33 & 20 & 36 & 28 & 48 & 38 & 62 \\
80 & 10 & 19 & 10 & 19 & 16 & 29 & 24 & 42 \\
100 & 2 & 4 & 0 & 0 & 4 & 8 & 20 & 36 \\
True stenosis area (\%) & & 32 & & 36 & & 41 & 58 & \\
\hline
\end{tabular}

D, diameter; A, area.

$\mathrm{D}$, diameter; $\mathrm{A}$, area. 
Fig. i1 Radiographs of barium filled glass tube $D$ in optimum horizontal plane to give maximum observed stenosis. (a) Incident $x$ ray beam $90^{\circ}$ to longitudinal axis of tube $\left(0^{\circ}\right.$ vertical rotation). (b) Incident $x$ ray beam $30^{\circ}$ to longitudinal axis of tube $\left(60^{\circ}\right.$ vertical rotation)). Percentage stenoses by diameter and corresponding cross sectional area at maximum point of constriction were calculated from observed diameters for two horizontal planes through varying degrees of vertical rotation and were compared with true cross sectional area stenosis as measured by planimetry for tube $D$.
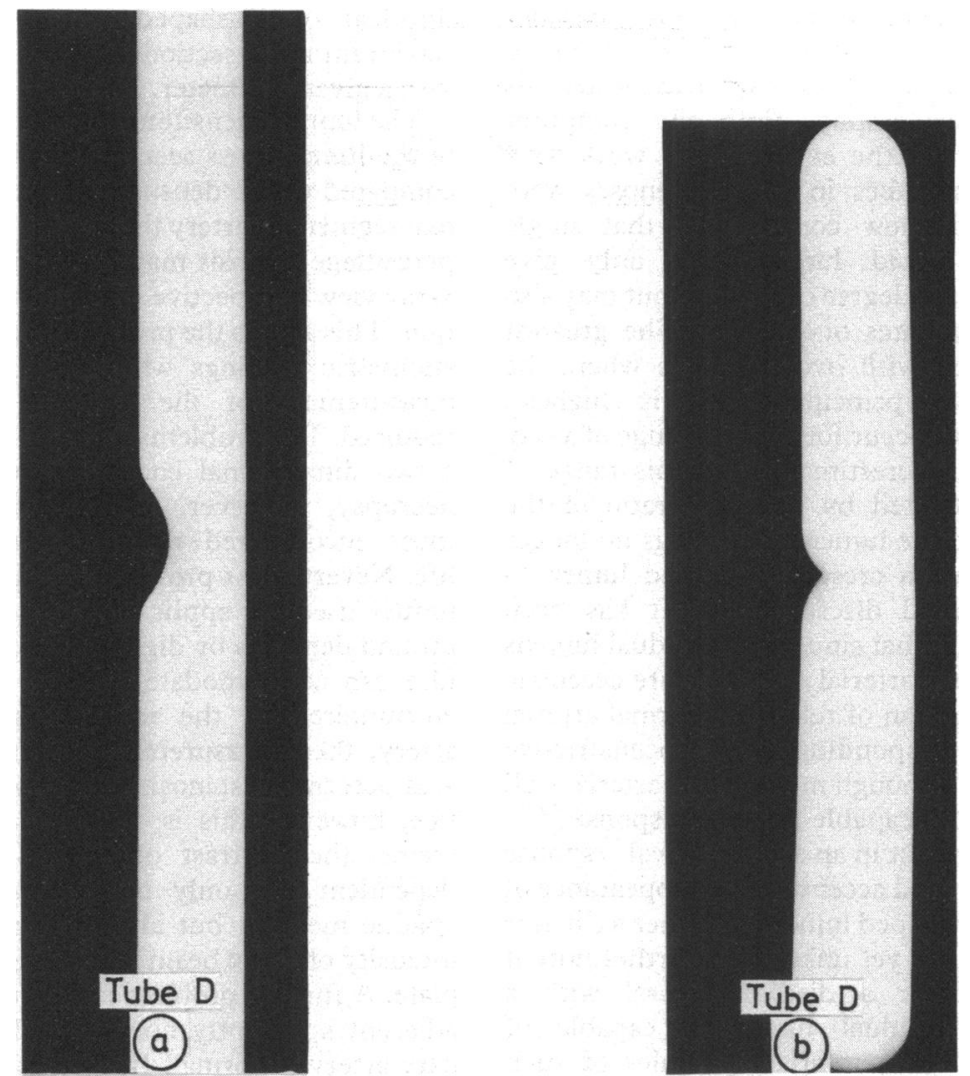

\begin{tabular}{|c|c|c|c|c|}
\hline \multirow[t]{2}{*}{ Vertical rotation } & \multicolumn{2}{|c|}{$\begin{array}{l}\text { Optimum horizontal rotational plane to give } \\
\text { maximum observed stenosis ( } \% \text { stenosis) }\end{array}$} & \multicolumn{2}{|c|}{$\begin{array}{l}\text { Horizontal rotational plane } 90^{\circ} \text { to optimum } \\
\text { plane (\% stenosis) }\end{array}$} \\
\hline & Diameter & Area & Diameter & Area \\
\hline $\begin{array}{l}0^{\circ} \\
20^{\circ} \\
40^{\circ} \\
60^{\circ} \\
\text { True stenosis area }(\%)\end{array}$ & $\begin{array}{l}58 \\
54 \\
53 \\
13 \\
\end{array}$ & $\begin{array}{l}82 \\
79 \\
78 \\
24 \\
58\end{array}$ & $\begin{array}{l}20 \\
19 \\
19 \\
18 \\
-\end{array}$ & $\begin{array}{l}36 \\
35 \\
34 \\
33 \\
58\end{array}$ \\
\hline
\end{tabular}

histological processing and light microscopy corrected any problem of luminal distortion which occurred for purely technical reasons. It is essential to decalcify the epicardial coronary arterial tree before transverse sectioning. Failure to do this may well result in gross distortion of the lumen in calcified arteries, the whole vessel wall effectively being crushed rather than sectioned.

By using perfusion-fixation, decalcification, and examination of transverse arterial segments with the dissecting microscope, we obtained results that accorded with those of Freudenberg and Lichtlen-that is although the lumens associated with uncomplicated atheromatous plaques were approximately circular most were elliptical or D-shaped and often eccentrically placed. We were able to confirm our suspicion that slit-like and crescentic shaped lumens were rare. Subsequent 
histological examination with the light microscope confirmed that such shapes indicated a polypoidal mass of acute mural thrombus which projected into the arterial lumen or massive intra-intimal thrombus formation which had occurred as a result of blood tracking into the intimal plaque via a fissure.

If crescentic and slit-like lumens are not a feature of uncomplicated atheromatous plaques, one must question whether small deviations from a circular lumen (elliptical or $\mathrm{D}$ shapes) are sufficient to explain underestimation of coronary arterial stenosis from clinical arteriography. Both the computer simulation study and the experimental work with barium filled glass tubes in which stenoses were artificially created show conclusively that single plane views of ovoid lumens not only give underestimates of the degree of stenosis but may also give gross overestimates of stenosis. The greatest discrepancies occur with ovoid lumens where the ratio of the two principal radii is highest. Underestimates will occur for a wider range of $x$-ray beam angles than overestimates and this range of beam angles is affected by both the ratio of the principal radii and the lumen shape. It is no longer correct to implicate a crescentic shaped lumen to explain the observed discrepancies. It has been suggested, however, that since most residual lumens in diseased coronary arterial segments are eccentric there must be a portion of relatively normal arterial wall capable of responding to vasoconstrictive agents during life, although much of the arterial wall will be fixed and incapable of any response. ${ }^{11} 12$ This feature may result in an asymmetrical response to vasoconstriction and accentuate the appearance of any elliptical or D-shaped lumen. Whether a slit-like lumen can result is as yet unknown. Furthermore it is debatable whether a diseased vessel with a centrally placed residual lumen is capable of vasoconstriction. The potential dynamics of such luminal shapes are well reviewed by Brown et al. ${ }^{1112}$

As regards interpretation of clinical arteriography in terms of the degree of arterial stenosis, the fact remains that errors will occur if only one plane of view is examined. Even if views in two planes are available the maximum stenosis observed is the usually accepted value but this in fact might represent an overestimation of the degree of stenosis. It is extremely difficult if not impossible to obtain more than one meaningful view of certain areas of the coronary artery anatomy. The problems are compounded when the incident $x$-ray beam is not perpendicular to the longitudinal axis of the vessel. Given that many residual lumens are non-circular, it follows that to report these in terms of percentage stenosis by diameter may be just as misleading. Equally, calculation of percentage stenosis by cross sectional area derived from diameters on the assumption that the lumen is circular may be just as meaningless. Histological assessment by cross sectional area based on lumen and vessel perimeters, assuming these to be circumferences of circles, is usually more accurate but will still give underestimates when the lumen is elliptical or D-shaped, since a circle has the maximum cross sectional area that can be obtained with a given perimeter.

The luminal densitometric readout relates directly to the lumen cross sectional area ${ }^{13-15}$ and if this is compared with a densitometric readout from a normal segment of artery then a true cross sectional area percentage stenosis may be obtained from only one $x$-ray view irrespective of the lumen shape and position. This avoids the problem of calibrating the densitometric readings which would be necessary if measurement of the absolute lumen area was required. The problems in being able to do this from a two dimensional coronary arteriogram taken at necropsy, however, are many-notwithstanding those encountered with angiograms taken during life. Nevertheless provided the image analysis techniques used are applicable to soft tissue and background densities by digital subtraction and an $x$-ray film can accommodate the full range of densities encountered in the radio-opaque contrast filled artery, then measurements of true cross sectional area percentage stenosis should be feasible. In practice, however, this is often more difficult than it seems, the contrast obtained in an $x$-ray being dependent not only on concentration of radioopaque medium but also on $x$-ray exposure time, intensity of $x$-ray beam, and developing of the $x$-ray plate. A further problem is the supposition that the adjacent apparently non-lesional segment of coronary artery is normal. In fact this is not always the case; regular diffuse narrowing may be entirely missed on $x$-ray so that if a severe stenosis is present within a long segment of less severe diffuse narrowing, the severe stenosis may still be underestimated. At present we have no idea of what the normal range of coronary artery calibre is for any given point within the epicardial coronary arterial tree. A definitive assessment must be to measure the flow rate beyond a stenosis, since the answer to "is $75 \%$ stenosis in a given vessel more important than $75 \%$ stenosis in a vessel twice its size?", begs the question as to what is the normal coronary artery diameter at any given point, and such a question can only be answered from flow studies.

The three dimensional reconstruction of the coronary arterial lumen from densitometric readings of two dimensional angiograms should be feasible pro- 
vided sufficient $x$-ray planes are examined. ${ }^{131416}$ If the stenosed lumen had a circular or ovoid shape then a limited number of views may well be adequate, provided that in this case some calibration is introduced. The more irregular the lumen shape, the greater the number of $x$-ray planes required to enable a reconstruction to be made. The importance of reconstruction may be to detect those lesions which are complicated by thrombosis or plaque fissuring or both and are therefore the more likely to precipitate sudden death or myocardial infarction.

\section{References}

1 Vlodaver Z, Edwards JE. Pathology of coronary atherosclerosis. Prog Cardiovasc Dis 1971; 14: 256-74.

2 Isner JM, Kishel J, Kent KM, Ronan JA Jr, Ross AM, Roberts WC. Accuracy of angiographic determination of left main coronary artery narrowing. Circulation 1981; 63: 1056-64.

3 Crawford T, Levene C. Medial thinning in atheroma. $f$ Pathol Bacteriol 1953; 66: 19-23.

4 Wolinsky H, Glagov S. Structural basis for the static mechanical properties of the aortic media. Circ Res 1964; 14: 400-13.

5 Hutchins GM, Bulkley BH, Ridolfi RL, Griffith LSC, Lohr FT, Piasio MA. Correlation of coronary arteriograms and left ventriculograms with post-mortem studies. Circulation 1977; 56: 32-7.

6 Glagov S, Zarins CK. Quantitating atheroslerosis. In: Bond MG, Insull W Jr, Glagov S, Chandler AB, Cornhill JF, eds. Clinical diagnosis of atherosclerosis. New York: Springer-Verlag, 1983: 11-35.

7 Zarins CK, Zatina MA, Glagov S. Correlation of post mortem angiography with pathologic anatomy. Quantitation of atherosclerotic lesions. In: Bond MG, Insull W Jr, Glagov S, Chandler AB, Cornhill JF, eds. Clinical diagnosis of atherosclerosis. New York: SpringerVerlag, 1983: 283-306.
8 Thomas AC, Davies MJ. The demonstration of cardiac pathology using perfusion-fixation. Histopathology 1985; 9: 5-19.

9 Thomas AC, Davies MJ. Post-mortem investigation and quantification of coronary artery disease. Histopathology 1985; 9: 959-76.

10 Freudenberg $\mathrm{H}$, Lichtlen PR. The normal wall segment in coronary stenosis - a post mortem study. $Z$ Kardiol 1981; 70: 863-69.

11 Brown BG. Coronary vasospasm. Observations linking the clinical spectrum of ischaemic heart disease to the dynamic pathology of coronary atherosclerosis. Arch Intern Med 1981; 141: 716-22.

12 Brown BG, Bobson EL, Dodge HT. Dynamic mechanisms in human coronary stenosis. Circulation 1984; 70: 917-22.

13 Reiber JHC, Troost GJ, Gerbrands JJ, Booman F, der Boer A, Serruys PW. Densitometric assessment severity of coronary obstruction from monoplane views; three-dimensional reconstruction arterial segment from two orthogonal views. In: Ripley KL, ed. Proceedings of IEEE Computer Society annual conference on computers in cardiology 1981. Long Beach, California: Computer Society, 1982: 333-6.

14 Reiber JHC, Gerbrands JJ, Kooijman CJ, et al. Computer-aided analysis of coronary obstructions from monoplane cineangiograms and three-dimensional reconstruction of an arterial segment from two orthogonal views. In: Short MD, ed. Proceeding of Hospital Physicists' Association conference of physical techniques in cardiological imaging 1982. Bristol: Adam Hilger, 1983: 173-88.

15 Crawford DW, Brooks SH, Barndt R Jr, Blankenhorn DH. Measurement of atherosclerotic luminal irregularity and obstruction by radiographic densitometry. Invest Radiol 1977; 12: 307-13.

16 Suetens P, Haegmans A, Oosterlinck A, Gybels J. An attempt to reconstruct the cerebral blood vessels from a lateral and a frontal angiogram. Pattern Recognition 1983; 16: 517-24. 